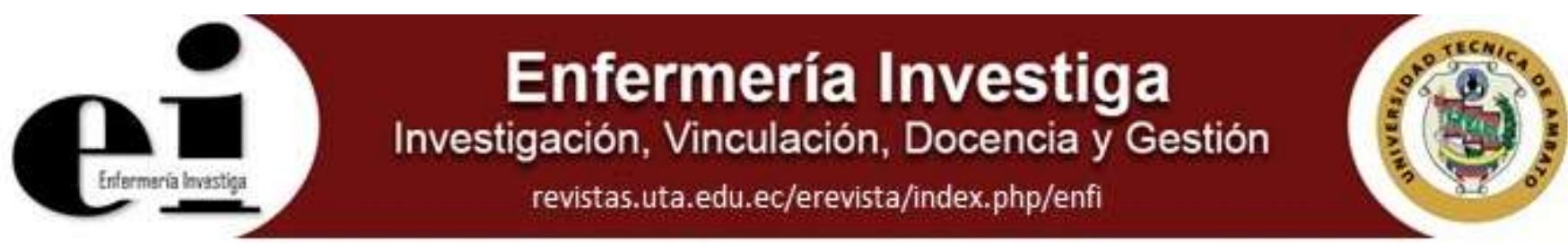

Artículo original

\title{
Síndrome de Burnout en estudiantes de la Universidad Técnica de Ambato, Ecuador Burnout Syndrome in students of Universidad Técnica de Ambato, Ecuador
}

Yury Rosales-Ricardo',2, Julio Mocha-Bonilla1 ${ }^{1}$, José Pedro Ferreira ${ }^{2}$

${ }^{1}$ Universidad Técnica de Ambato, Ambato, Ecuador.

2 Universidad de Coimbra. Portugal

Rosales-Ricardo Y., Mocha-Bonilla J., Ferreira J.P. Síndrome de Burnout en estudiantes de la Universidad Técnica de Ambato, Ecuador. Enferm Inv. 2020; 5(2)3741

2477-9172 / 2550-6692 Derechos Reservados ( 2020 Universidad Técnica de Ambato, Carrera de Enfermería. Este es un artículo de acceso abierto distribuido bajo los términos de la Licencia Creative Commons, que permite uso ilimitado, distribución y reproducción en cualquier medio, siempre que la obra original es debidamente citada.

\section{Historia:}

Recibido: 05 enero 2020

Revisado: 10 febrero 2020

Aceptado: 27 febrero 2020

Palabras Claves: Salud mental, síndrome de burnout, estrés, correlación, estudiantes.

Keywords: Mental health, burnout syndrome, stress, correlation, students

\section{Resumen}

Introducción: El síndrome de burnout es un problema de salud mental de gran repercusión social en nuestros días en los estudiantes universitarios. Objetivo: determinar los niveles de Síndrome de Burnout en estudiantes de la Universidad Técnica de Ambato, Ecuador.

Material y métodos: Estudio descriptivo transversal. La muestra fue probabilística con muestreo estratificado con participación proporcional. Se utilizó el Maslach Burnout Inventory Students Survey.

Resultados: agotamiento: ítem de mayor valor el $2(3,68 \pm 1,65)$, el más bajo el $4(2,24 \pm 1,58)$. Cinismo: el $14(1,87 \pm 1,31)$ y el $8(1,13 \pm 1,53)$ respectivamente. En eficacia el $12(4,89 \pm 1,29)$ y el $7(3,70 \pm 1,46)$. En estrés el $12(2,52 \pm 1,08)$ y el $6(1,59 \pm 1,00)$ respectivamente. Más del $28 \%$ de los estudiantes diagnosticados con niveles altos y moderados de agotamiento, casi el $19 \%$ en los mismos niveles de Cinismo. El Agotamiento dio niveles sobre el $10 \%$. Casi la mitad de no tenía cinismo y el 32 \% tenía niveles bajos. Las Facultades con más prevalencia del SB fueron las Ciencias Humanas y de la Educación y la de Ciencias de la Salud. La de menor prevalencia de SB fue Jurisprudencia y Ciencias Sociales.

Conclusiones: El agotamiento fue la dimensión de mayor prevalencia. El sexo femenino fue el más afectado. La mayoría de los estudiantes fueron diagnosticados con niveles bajos y moderados.

\begin{abstract}
Introduction: Burnout syndrome is a mental health problem of great social impact on university students today.

Objective: to determine the levels of Burnout Syndrome in students of the Technical University of Ambato, Ecuador.

Material and methods: Cross-sectional descriptive study. The sample was probabilistic with stratified sampling with proportional participation. The Maslach Burnout Inventory Students Survey was used.

Results: exhaustion: highest value item $2(3,68 \pm 1,65)$, lowest value $4(2,24 \pm 1,58)$. Cynicism: $14(1,87 \pm 1,31)$ and $8(1,13 \pm 1,53)$ respectively. In efficiency $12(4,89 \pm 1,29)$ and $7(3,70 \pm 1,46)$. In stress $12(2,52 \pm 1,08)$ and $6(1,59 \pm 1,00)$ respectively. More than $28 \%$ of the students diagnosed with high and moderate levels of exhaustion, almost $19 \%$ in the same levels of Cynicism. Exhaustion gave levels above $10 \%$. Almost half had no cynicism and $32 \%$ had low levels. The Faculties with the highest prevalence of SB were the Human and Education Sciences and Health Sciences. The lowest prevalence of SB was Jurisprudence and Social Sciences.

Conclusions Exhaustion was the dimension with the highest prevalence. The female sex was the most affected. Most students were diagnosed with low and moderate levels.
\end{abstract}




\section{Introducción}

En estudiantes universitarios, una buena salud mental es esencial para un óptimo desarrollo en esta etapa de su vida. Pues iniciar el estudio de una carrera profesional puede ser una fuente de tensión que acecha el bienestar psicológico, el cual se puede resultar comprometido en el aprovechamiento académico, en la salud física o en la salud mental (1-13).

Los estudiantes universitarios experimentan cada vez más problemas de salud mental que afectan a su rendimiento, su bienestar y sus vidas en general. Las exigencias académicas, ostensiblemente más acentuadas que las enfrentadas en la vida escolar, podrían estar entre los factores más tensores, además de la presión académica propia de la vida universitaria se da a partir de un cambio considerable en relación al sistema de enseñanza escolar, en el que los jóvenes son mucho menos autónomos (14-17).

El Síndrome de Burnout (SB) en estudiantes se define como una respuesta emocional negativa, persistente, relacionada con los estudios, formada por una sensación de encontrarse exhausto, de no poder más en las tareas como estudiante (agotamiento); una actitud cínica o de distanciamiento con el significado y la utilidad de los estudios que se están realizando (cinismo); y un sentimiento de incompetencia académica como estudiante (ineficacia) (18, 19), agotamiento por las demandas del estudio, además de actitudes de desinterés, autosabotaje frente a las actividades académicas y dudas acerca del valor del estudio, además de sentimientos de incompetencia como estudiante (20-22).

En el Ecuador se han encontrado muy pocos estudios de prevalencia de ambos determinantes de la mala salud mental, y mucho menos en esta importante población de estudiantes universitarios que puedan permitir un correcto diagnóstico, prevención y tratamiento en ambos casos. Por tanto, el objetivo del estudio fue determinar los niveles de Síndrome de Burnout y Estrés Percibido en estudiantes universitarios del Ecuador.

\section{Método}

Se realizó un estudio descriptivo transversal. Donde se determinaron los niveles de Síndrome de Burnout y Estrés percibido en estudiantes universitarios ecuatorianos.

\section{Muestra}

La muestra se identificó en octubre de 2018, partió de la población de estudiantes de la Universidad Técnica de Ambato, universidad pública del Ecuador, la muestra fue probabilística y se utilizó un muestreo estratificado con participación proporcional. Se sustrajeron muestras representativas de forma aleatoria simple de cada estrato de todos los años y de ambos sexos de todas las carreras de todas las facultades.

El tamaño de la muestra se obtuvo partiendo del error estándar de la distribución muestral de proporciones y del valor crítico $\mathrm{K}$, correspondiente al nivel de confianza elegido. El método de muestreo que se considera más acertado es el aleatorio estratificado que permite una representatividad de la población y la muestra calculada: para $\mathrm{N}=13000$; la muestra mínima calculada es de $n=374$ para un nivel de Confianza del $95 \%$, margen de error del $5 \%$. Este valor se multiplicó por 5 dando $n=1870$. De ahí se excluyeron 179 por diferentes causas. Para quedar una $n=1691$. Finalmente hubo una pérdida de 91 y la muestra final para los datos disponibles fue $n=1600$.

Variables e Instrumentos de estudio:

Síndrome de Burnout en estudiantes: se utilizó el Maslach Burnout Inventory Students Survey (MBI-SS).

Se estructura en 3 dimensiones divididos en 15 ítems: Agotamiento emocional (Con los ítems 1, 2, 3, 4 y 6 . Por ejemplo, «Estoy cansado cuando me levanto por la mañana y tengo que afrontar otro día en la universidad»), Cinismo (Con los ítems 8, 9, 13 y 14. Por ejemplo, «Dudo de la trascendencia y valor de mis estudios») y Eficacia académica (Con los ítems 5, 7, 10,11, 12 y 15. Por ejemplo, «Creo que contribuyo efectivamente durante las clases en la universidad»). La escala de respuesta es tipo Likert y oscila entre 0 («nunca») y 6 («siempre»).

Para la evaluación de los resultados se adaptó el baremo propuesto por el Instituto Nacional de Seguridad e Higiene en el Trabajo (23) y utilizado para estudiantes por Hederich-Martínez et al. (24):

Agotamiento: Sin SB: menos de 1.2; Bajo: 1.3 - 2.0; Moderado: 2.1 - 2.8; Alto 2.9 o más. Cinismo: Sin SB: menos de 0.5; Bajo: 0.6 - 1.24; Moderado: 1.25 - 2.25; Alto: 2.26 o más. Eficacia: Sin SB: menos de 2.83; Bajo: 2.83 3.83; Moderado: 3.84 - 5.16; Alto: 5.17 o más. 


\section{Procedimientos e intervención}

Después de conocer la muestra de estudio se les aplicó el MBI-SS a los estudiantes luego de tener su consentimiento informado.

A los participantes se les garantizó el anonimato y la confidencialidad de la información proporcionada. En la hoja de consentimiento informado se indicaba si se deseaba o no participar, marcando la opción correspondiente, y se firmaba. El cuestionario, entregado posteriormente, no solicitaba datos personales de identificación, manteniendo así el anonimato del participante. En la base de datos cada caso quedó identificado con un número.

Los cuestionarios se distribuyeron a lo largo de septiembre y octubre de 2018. Su entrega fue de manera individual, con una breve explicación del objetivo de la investigación que se estaba desarrollando, y la garantía del anonimato y la confidencialidad de las respuestas. Se destacó su accesibilidad exclusiva para los miembros del equipo, para fines de investigación.

Tratamiento estadístico de datos

La totalidad de los datos recogidos fueron analizados a través del programa informático SPSS "Statistical Package for the Social Sciences, version 25.0 for Windows". Estadística descriptiva (media, desviación estándar, p value y cálculo porcentual).

La significancia estadística ( $p$ value) se determinó mediante la prueba de Kolmogorov-Smirnov para una muestra.

\section{Resultados}

En el caso del Síndrome de Burnout en su dimensión Agotamiento los ítems de mayor valor en su media fueron el 2 (Me encuentro agotado físicamente al final de un día de universidad) y el de media más baja fue el ítem 4 (Estudiar o ir a clases todo el día es una tensión para mí). En la dimensión Cinismo el ítem de media más alta fue el 14 (Me he distanciado de mis estudios porque pienso que no serán realmente útiles) y el más bajo fue el 8 (He perdido interés en la carrera desde que empecé la universidad). Y finalmente, en la dimensión Eficacia Académica el de mayor valor de media fue el ítem 12 (He aprendido muchas cosas interesantes durante mi carrera) y el de más baja media fue el 7 (Creo que contribuyo efectivamente con las clases en mi universidad).

En los resultados generales, más del $28 \%$ de los estudiantes fueron diagnosticados con niveles altos y moderados de agotamiento emocional, y casi el $19 \%$ en los mismos niveles de Cinismo la principal dimensión del SB. El Agotamiento fue de todas las dimensiones del SB la que más altos niveles dio, sobre el $10 \%$, aunque la mayoría de los estudiantes fueron diagnosticados con niveles bajos y moderados de este Síndrome. En la dimensión Cinismo casi la mitad de no tenía SB y el 32 \% tenía niveles bajos. En tanto la percepción del estrés primó significativamente la no existencia de estrés percibido, sobre niveles de percepción moderados y altos, pues estos dos juntos alcanzaron a poco más del $11 \%$ del total (ver tabla 1$)$.

Tabla 1. Resultados Generales por dimensión y variable $(n=1600)$

\begin{tabular}{ccccc}
\hline & Sin SB (\%) & Bajo (\%) & Moderado (\%) & Alto (\%) \\
\hline $\begin{array}{c}\text { Agotamiento } \\
\text { SB }\end{array}$ & $731(45.69 \%)$ & $408(25.50 \%)$ & $298(18.63 \%)$ & $163(10.19 \%)$ \\
\hline Cinismo SB & $787(49.19 \%)$ & $511(31,94 \%)$ & $188(11.75 \%)$ & $114(7.13 \%)$ \\
\hline EA SB & $905(56.56 \%)$ & $501(31.31 \%)$ & $109(6.81 \%)$ & $85(5.31)$ \\
\hline
\end{tabular}

En el resultado por facultades las que más números de estudiantes tuvieron niveles altos del SB fueron las Facultades de Ciencias Humanas y de la Educación y la de Ciencias de la Salud. En cambio, las de menos estudiantes con el SB y niveles más bajos de éste fueron en las Facultades de Jurisprudencia y Ciencias Sociales y en Agropecuarias. El sexo femenino fue el más afectado, pues hubo mayor porcentaje de mujeres con niveles altos y moderados de SB que de hombres. En cambio, en la percepción de estrés hubo una gran paridad en cuanto a los porcientos.

Como el valor de p fue menor o igual que 0,05 se rechaza la hipótesis nula y se concluye que hay evidencias suficientes para pensar que la muestra proviene de la distribución especificada, con un nivel de significación del $5 \%$. 


\section{Discusión}

En un estudio realizado en estudiantes de la carrera de Licenciatura en Enfermería, de Escuela de Enfermería de la Universidad de Costa Rica, el 18,8\% de la población estudiada presenta el SB, niveles un poco más bajos que los encontrados en nuestro estudio $(22.63 \%$ y $37.2 \%$ en alto y moderado respectivamente) se evidencia que el síndrome se presenta más en la población de primer año $(32,2 \%)$, seguida por la de quinto año (29, $1 \%)$.son las mujeres las que más presentan el síndrome (un 22,7\%) (25).

En 56 estudiantes de medicina de la Universidad Autónoma de México un 27\% mostró ineficacia; un 11\%, cinismo, y un $7 \%$, agotamiento. La prevalencia de dos dimensiones fue del $5 \%$. Se encontraron diferencias significativas $(p<$ $0,05)$ para la relación con el grupo, tocar un instrumento, cantar o actuar, sitio de residencia y la violencia en el traslado. La dimensión más frecuente fue ineficacia $(27 \%)$, seguida de cinismo $(11 \%)$ y agotamiento $(7 \%)$, en nuestro estudio hubo algunas coincidencias en cuanto a sus resultados (26).

Arias \& Gutiérrez (27) determinaron la prevalencia y factores asociados al síndrome de burnout, en estudiantes internos de medicina del Hospital Militar Central de Lima, Perú. Y encontraron una prevalencia de síndrome de burnout solo en cinco internos (5,2\%). Se evidenció que el 27,1\% (26 internos) presentaba niveles altos de agotamiento emocional. En 13 de ellos (13,5\%) se presentaron niveles altos despersonalización y en 20 (20,8 \%), se identificó una realización personal baja.

Merchán-Galvis et al. (28) determinaron la prevalencia del Síndrome de Burnout en los estudiantes de Medicina de la Universidad Icesi en Cali, Colombia. Ésta fue del 4,3\%, mucho más baja que la encontrada en nuestra investigación, destacando que el $47,8 \%$ de los estudiantes presentó reducida realización personal. El X semestre se asoció con alteraciones significativas en todas las dimensiones valoradas. El Síndrome de Burnout estaba presente en el $4,3 \%(n=7)$ de los estudiantes, según la interpretación del instrumento al obtener una puntuación alta en las dimensiones de AE y DP junto a una puntuación baja en la dimensión de RP. Adicionalmente, el grupo investigador consideró como limítrofe o probable los estudiantes que presentaron dos de las tres dimensiones afectadas, encontrándose que el $21,1 \%(n=34)$ estaba en esta situación.

En estudiantes residentes de especialidades médicas de una universidad pública en el departamento del CaucaColombia, la razón de prevalencia del síndrome de Burnout fue del 0,9 y estuvo presente en el $47,5 \%$ de los participantes, El $42,5 \%$ presentaban cansancio emocional, el $55 \%$ presentaron baja despersonalización y solo la mitad reportaron estar en un alto nivel de realización personal, y presentan los puntajes asignados a las variables resultantes del test de Burnout, observando que para el cansancio emocional en dos extremos (bajo y alto) se encuentran los mayores porcentajes con un $42,5 \%$ respectivamente, mientras que en despersonalización el $55 \%$ lo presenta en calificación baja, el $50 \%$ es calificado en alto para realización personal y el $47,5 \%$ presenta riesgo de Burnout. No siendo coincidente con nuestros resultados (29).

La población de 155 estudiantes de Psicología de la Universidad Veracruzana y 101 de la Licenciatura en Administración del Instituto Tecnológico de Veracruz, un total de 256 se encontró con nivel alto de burnout estudiantil un $1.29 \%$ en mujeres de la licenciatura en administración, y un $0 \%$ en los hombres, en cuanto a la licenciatura en psicología ni hombres ni mujeres presentaron nivel alto de burnout estudiantil al contrario de nuestro estudio. (30).

\section{Conclusiones}

Más del $28 \%$ de los estudiantes fueron diagnosticados con niveles altos y moderados de agotamiento emocional, y casi el $19 \%$ en los mismos niveles de Cinismo la principal dimensión del SB. El Agotamiento fue de todas las dimensiones del SB la que más altos niveles dio, sobre el $10 \%$. En la dimensión Cinismo casi la mitad de no tenía $\mathrm{SB}$ y el $32 \%$ tenía niveles bajos. Las facultades que más números de estudiantes tuvieron niveles altos del SB fueron las Facultades de Ciencias Humanas y de la Educación y la de Ciencias de la Salud. En cambio, las de menos estudiantes con el SB y niveles más bajos de éste fueron en las Facultades de Jurisprudencia y Ciencias Sociales y en Agropecuarias. El sexo femenino fue el más afectado, pues hubo mayor porcentaje de mujeres con niveles altos y moderados de SB que de hombres.

\section{Conflicto de intereses}

Ninguno declarado por los autores.

\section{Financiación}

Autofinanciado. 


\section{Referencias}

1. García, Elena. La evaluación por competencias en la educación superior. Profesorado. Revista de curriculum y formación de profesorado. 2008,12 (3): 1-16.

2. Guzmán, Maricela Osorio, Santa Perrello, and Carlos Prado Romero. Burnout académico en una muestra de estudiantes universitarios mexicanos. Enseñanza e Investigación en Psicología 2.1 (2020): 27-37.

3. Ramírez, Leo. Estudio y desarrollo de las competencias emocionales en estudiantes de medicina. Una aproximación bibliométrica. Investigación en educación médica. 2019; 8(3): 92-102.

4. Sevrain-Goideau, Marion. Forum theater staging of difficult encounters with patients to increase empathy in students: evaluation of efficacy at The University of Angers Medical School. BMC Medical Education. 2020:1-8.

5. Chen, Christine C. Professional Quality of Life among Occupational Therapy Practitioners: An Exploratory Study of Compassion Fatigue. Occupational Therapy in Mental Health. 2020:1-14.

6. Janighorban, Mojgan. La correlación entre el empoderamiento psicológico y el agotamiento del empleo en parteras que trabajan en la sala de partos de hospitales. Iranian Journal of Nursing and Midwifery Research. 2020: 128-37

7. Son, Hyeong-jun, and Sung-cheol Jung. The Effect of Social Support on Job Satisfaction and the Mediating Role of Burnout. Journal of Venture Innovation. 2019:81-92.

8. Lopes, Tátila Martins. Vaz de Carvalho, Anelisa. Terapia Cognitivo-Comportamental na síndrome de Burnout: contextualização e intervenções. Sinopsys, Boletim-Academia Paulista de Psicologia. 2019:289-290.

9. Lopes, Maxime, and Robert J. Vallerand. The role of passion, need satisfaction, and conflict in athletes' perceptions of burnout. Psychology of Sport and Exercise. 2020:1016-1074.

10. Cova, F., Alvial, W., Aro, M., Bonifetti, A., Hernández, M. y Rodríguez, C. Problemas de salud mental en estudiantes de la universidad de Concepción. Terapia psicológica, 2007. 25:105-112

11. Webber, Ashleigh. "NHS-wide interventions needed to tackle 'endemic'burnout." Occupational Health \& Wellbeing. 2019: 5-5.

12. Kwiatkowska-Ciotucha, Dorota. Occupational Burnout in Health Care-Analysis of Systemic and Organisational Risks as Well as Possible Preventive Actions." Econometrics. 2019:43-62.

13. Yew, Victor Goh Weng, and Hazel Melanie Ramos. The relationship between perceived organizational support, age, and burnout: Implications for the Malaysian higher education workplace. International Journal of Employment Studies. 2019, 27(2):35.

14. Trigozo, Luis Fernando Chunga, et al. "Relación entre estrés laboral y Síndrome de Burnout en conductores de taxi de la ciudad de Trujillo. Perú. Vallejian Medical Journal. 2020; 9(1):13-17.

15. Pellerone, Monica. Burnout and Self-Perceived Instructional Competence: An Exploratory Study of a Group of Italian Female Elementary School Teachers. International Journal of Environmental Research and Public Health. 2020; 17(4):1356.

16. Harvey, Felicia. Physician Burnout Quality of Life/Wellness Resource Pilot Program. Journal of Medical Research and Health Sciences. 2020; 3(2):886912.

17. Bocheliuk, Vitalii Y. Emotional Burnout: Prevalence Rate and Symptoms in Different Socio-Professional Groups. Journal of Intellectual Disability-Diagnosis and Treatment. 2020; 8(1):33-40.

18. Dallacosta, F. M. "Stress and burnout syndrome in health professionals. Int J Fam Commun Med. 2019; 3(5):179-183

19. Obadić, Petra, and Ivan Mlakar. Burnout syndrome in healthcare workers. Hrana u zdravlju i bolesti: znanstveno-stručni časopis za nutricionizam i dijetetiku. Štamparovi dani. 2019 (11):37

20. Fauzia, Liza, Kadek Ayu Erika, and Andi Masyitha Irwan. Literature study: validity and reliability test of maslach instruments burnout inventory-human services survey (mbi-hss) in nurses in several countries. Jurnal Ilmu Keperawatan: Journal of Nursing Science. 2020; 7(2):160-166.

21. Cheng, Michelle Y. Exploration of Mistreatment and Burnout Among Resident Physicians: a Cross-Specialty Observational Study. Medical Science Educator. 2020):1-7

22. Treviño, E. Burnout en las pymes: una aproximación desde el nivel gerencial." AD-minister. 2019;35:137-163.

23. Instituto Nacional de Seguridad e Higiene en el Trabajo. Archivos de Prevención de Riesgos Laborales.2000; 19(2): $124-126$.

24. Hederich-Martínez, C., \& Caballero-Domínguez, C. C. Validación del cuestionario Maslach Burnout Inventory-Student Survey (MBI-SS) en contexto académico colombiano. CES Psicología. 2016; 9(1):1-15.

25. Reyes, N. B., \& Blanco, N. R. Prevalencia del Síndrome de Burnout académico en el estudiantado de Enfermería de la Universidad de Costa Rica. Enfermería Actual en Costa Rica. 2016, (31).

26. Plett-Torres, Tanya, Martínez-Flisser, Gina, Gutiérrez-Barreto, Samuel E, Vives-Varela, Tania, Hamui-Sutton, Alicia, \& Flisser, Ana. Burnout en estudiantes del Plan de Estudios Combinados en Medicina, Facultad de Medicina, Universidad Nacional Autónoma de México. FEM: Revista de la Fundación Educación Médica, 2018; 21(6): 295-303.

27. Arias, Renzo, \& Gutiérrez, Ericson L. Prevalencia del síndrome de burnout en internos de medicina del Hospital Militar Central de Lima. Revista Cubana de Medicina Militar, 2018; 47(4).

28. Merchán-Galvis, Ángela María, Albino Matiz, Angie Yicenia, Bolaños-López, Jhon Edwar, Millán, Nicolás, \& Arias-Pinzón, Ary Andrés. Síndrome de Burnout y factores asociados en estudiantes de Medicina. Educación Médica Superior. 2018; 32(3):172-180.

29. Jácome, S. J., Villaquiran-Hurtado, A. F., García, C. P., \& Duque, I. L. Prevalencia del síndrome de Burnout en residentes de especialidades médicas. Revista Cuidarte. 2019; 10(1).

30. Barradas Alarcón, ME, Trujillo Castro, PG, Sánchez Barradas, AV, \& López González, J. Burnout estudiantil en universitarios veracruzanos. RIDE. Revista Iberoamericana para la Investigación y el Desarrollo Educativo. 2017; 7(14):15-33.

Yury Rosales-Ricardo. Orcid: https://orcid.org/0000-0002-0525-2405

Julio Mocha-Bonilla: Orcid:https://orcid.org/0000-0002-8903-4746

José Pedro Ferreira. Orcid:https://orcid.org/0000-0002-4427-327 